\title{
EL REGISTRO DE DOCUMENTALES Y NOTICIARIOS, UNA OPORTUNIDAD PARA INCREMENTAR LA DOCUMENTACIÓN DEL PATRIMONIO HISTÓRICO EDUCATIVO $^{1}$
}

\author{
Eulàlia Collelldemont Pujadas \\ Universidad de Vic - Universidad Central de Catalunya \\ eulalia@uvic.cat \\ Núria Padrós-Tuneu \\ Universidad de Vic - Universidad Central de Catalunya \\ nuria.padros@uvic.cat \\ Pilar Prat Viñolas \\ Universidad de Vic - Universidad Central de Catalunya \\ pilar.prat@uvic.cat
}

\section{RESUMEN}

En el artículo se presenta el registro de las producciones audiovisuales de documentales y noticiarios producidos entre 1940 y 1975 realizado desde el MUVIP (Museo Virtual de Pedagogía), la finalidad con la que se ha realizado y el proceso de toma de decisiones durante la elaboración del mismo. Por otra parte, se presenta un primer análisis serial realizado a partir del registro que nos permite conocer con qué frecuencia aparecen las dimensiones y palabras clave y, de esta forma, esbozar un primer análisis que nos permite confirmar algunas hipótesis previas o aspectos no previstos en el proyecto de investigación ARAEF: Análisis de las representaciones audiovisuales de la educación en documentales y noticiarios durante el franquismo. Asimismo, consideramos que los registros o inventarios son herramientas muy útiles para desarrollar investigaciones temáticas, así como comparativas diacrónicas. Su elaboración es una tarea básica en la realización de proyectos sobre el patrimonio histórico educativo.

Palabras clave: Noticiarios. Franquismo. Patrimonio histórico educativo.

\section{THE REGISTRATION OF DOCUMENTARIES AND NEWS, AN OPPORTUNITY TO INCREASE THE DOCUMENTATION OF THE EDUCATIONAL HERITAGE}

\begin{abstract}
The article presents the register of the audiovisual productions of documentaries and newreels produced between 1940 and 1975 made from the MUVIP (Virtual Museum of Pedagogy), the purpose for which it was made and the decision-making process during the preparation of the same. Moreover, we present a first serial analysis made using the register that allows us to know how often the dimensions and keywords appear and, in this way, to sketch a first analysis that allows us

\footnotetext{
${ }^{1}$ Este artículo se ha realizado en el marco del proyecto "Análisis de las representaciones audiovisuales de la educación en documentales y noticiarios durante el franquismo" del Programa Estatal de Investigación, Desarrollo e Innovación Orientada a los Retos de la Sociedad, en el marco del Plan Estatal de Investigación Científica y Técnica y de Innovación 2013-2016, (Ref. EDU2017-89646-R, AEI/FEDER, UE).
}

Rev. Iberoam. Patrim. Histórico-Educativo, Campinas (SP), v. 05, p. 1-18, e019024, 2019. 
to confirm some previous hypotheses and, also, aspects not foreseen in the ARAEF research project: Analysis of the audiovisual representations of education in documentaries and news programs during the Franco regime. We consider that registers or inventories are very useful tools for developing thematic research, as well as diachronic comparisons. Its elaboration is a basic task in the course of developing projects on the historical educational heritage.

Keywords: Newsreels. Francoism. Historic Educational heritage.

\section{O REGISTRO DE DOCUMENTÁRIOS E NOTÍCIAS, UMA OPORTUNIDADE PARA ACRESCENTAR A DOCUMENTAÇÃO DO PATRIMÔNIO HISTÓRICO EDUCATIVO}

\section{RESUMO}

$\mathrm{O}$ artigo apresenta o registro das produções audiovisuais de documentários e notícias produzidas entre 1940 e 1975, feitas no MUVIP (Museu Virtual de Pedagogia), a finalidade para a qual foram feitas e o processo de tomada de decisão durante a preparação do o mesmo. Por outro lado, uma primeira análise serial é realizada com base no registro que nos permite saber com que frequência as dimensões e as palavras-chave aparecem e, assim, esboçar uma primeira análise que nos permite confirmar algumas hipóteses ou aspectos anteriores não previstos em o projeto de pesquisa da ARAEF: análise das representações audiovisuais da educação em documentários e programas de notícias durante o regime de Franco. Também consideramos que registros ou inventários são ferramentas muito úteis para o desenvolvimento de pesquisas temáticas, bem como comparações diacrônicas. Sua elaboração é uma tarefa básica na realização de projetos sobre o patrimônio histórico educativo.

Palavras-chave: Noticiários. Franquismo. Patrimônio Histórico Educativo.

\section{LE REGISTRE DE DOCUMENTAIRES ET DE NOUVELLES, UNE OPPORTUNITÉ DE AUGMENTER LA DOCUMENTATION DU PATRIMOINE HISTORIQUE ÉDUCATIF}

\section{RÉSUMÉ}

Cet article présente le registre des productions audiovisuelles de documentaires et les programmes d'actualités produits entre 1940 et 1975 par le MUVIP (Musée Virtuel de la Pédagogie), le but pour lequel il a été créé et le processus de décision lors de leur élaboration. D'autre part, nous présentons une première analyse en série réalisée à l'aide du registre, qui nous permet de connaître la fréquence d'apparition des dimensions et des mots-clés et, ainsi, d'esquisser une première analyse permettant de confirmer certaines hypothèses antérieures ainsi que des aspects non prévus dans le projet de recherche ARAEF: Analyse des représentations audiovisuelles de l'éducation dans les documentaires et les programmes d'actualités sous le régime franquiste. Nous considérons que les registres ou les inventaires sont des outils très utiles pour développer une recherche thématique, ainsi que des comparaisons diachroniques. Son élaboration est une tâche fondamentale dans la réalisation de projets sur le patrimoine éducatif historique.

Mots-clés: Actualités. Franquisme. Patrimoine historique éducatif.

Rev. Iberoam. Patrim. Histórico-Educativo, Campinas (SP), v. 05, p. 1-18, e019024, 2019. 


\section{INTRODUCCIÓN}

El registro de los bienes documentales y materiales ha mantenido la atención de los distintos archivos y museos pedagógicos. Inicialmente, las formas de registrar los bienes eran consideradas una cuestión museológica interna, y por esta razón, cada institución desarrollaba su ficha técnica con campos más o menos parecidos. Esta situación era aún más habitual en los museos pedagógicos, básicamente por dos razones: su origen singular al margen de las redes museísticas generales, muchos eran fruto de iniciativas particulares de inspectores, o bien de colectivos municipales o, en otros casos, de grupos de investigación universitaria, como puede observarse en la obra colectiva coordinada por Pablo Álvarez de 2016, Los Museos Pedagógicos en España. Entre la memoria y la creatividad, y también por las funciones inicialmente adjudicadas a los centros de conservación del patrimonio histórico educativo. Por todo ello, a dichos centros, se les puede aplicar perfectamente el diagnóstico de la situación museística general en España comentado por Eva Ma Alquézar Yañez, de la Subdirección General de Museos Estatales en el año 2004:

El proyecto de Normalización Documental de Museos Estatales partió de un informe de diagnóstico de la situación documental de los museos de gestión exclusiva de la SGME elaborado en 1993. Este informe presentaba un estado de la cuestión definido por la disparidad de situaciones, la diversidad de sistemas de trabajo y la falta de criterios comunes, disparidad que aumentaba si se extendía el estudio a museos fuera del ámbito de gestión de la SGME [...].

Entre las circunstancias que se apuntaban como causas de esta situación podemos citar: las particularidades de la historia de cada uno de los museos; el predominio de la investigación científica sobre otras funciones de los museos, y, en este sentido, la particularización de los sistemas documentales en relación con la disciplina científica predominante en la investigación de cada museo; la falta de análisis global de la mecánica del trabajo museístico; la carencia de un concepto claro de servicio público, que sí han tenido instituciones similares del ámbito del patrimonio, como las bibliotecas; o la falta de rentabilidad económica de una normalización de reglas catalográficas, que sí existe en el ámbito de la biblioteconomía. (p. 31).

Bien es cierto que, si analizamos, por ejemplo, los datos recogidos sobre la documentación museística general realizada en el año 2017 por el Departament de Cultura de Catalunya vemos que el proceso de inventariar los bienes de los museos, presenta aún bastantes deficiencias, especialmente en aquellos museos más pequeños, como suele ser el caso de los museos pedagógicos:

Rev. Iberoam. Patrim. Histórico-Educativo, Campinas (SP), v. 05, p. 1-18, e019024, 2019. 
Tabla 1- Actividad de documentación de las colecciones.

\begin{tabular}{|l|l|}
\hline Grandes museos & \\
\hline Número de fichas de inventario general realizadas en 2015 & 52.224 \\
\hline Número de fichas de inventario general revisadas en 2015 & 13.547 \\
\hline Número de fichas de inventario general digitalizadas en 2015 & 69.068 \\
\hline Museos de medio tamaño & \\
\hline Número de fichas de inventario general realizadas en 2015 & 16.687 \\
\hline Número de fichas de inventario general revisadas en 2015 & 15.031 \\
\hline Número de fichas de inventario general digitalizadas en 2015 & 12.190 \\
\hline Museo de medio o pequeño tamaño & \\
\hline Número de fichas de inventario general realizadas en 2015 & 4.484 \\
\hline Número de fichas de inventario general revisadas en 2015 & 5.683 \\
\hline Número de fichas de inventario general digitalizadas en 2015 & 7.413 \\
\hline Pequeños museos & \\
\hline Número de fichas de inventario general realizadas en 2015 & 873 \\
\hline Número de fichas de inventario general revisadas en 2015 & 11.699 \\
\hline Número de fichas de inventario general digitalizadas en 2015 & 3.777 \\
\hline Museos al límite de la sostenibilidad & \\
\hline Número de fichas de inventario general realizadas en 2015 & 321 \\
\hline Número de fichas de inventario general revisadas en 2015 & 1.324 \\
\hline Número de fichas de inventario general digitalizadas en 2015 & 976 \\
\hline Total de museos & \\
\hline Número de fichas de inventario general realizadas en 2015 & 74.589 \\
\hline Número de fichas de inventario general revisadas en 2015 & 42.284 \\
\hline Número de fichas de inventario general digitalizadas en 2015 & 93.424 \\
\hline $\begin{array}{l}\text { Destaca además que solo el 52\% de los museos tiene el 100\% de los } \\
\text { bienes documentados y 29 entre el 80\% y 100\%. }\end{array}$ \\
\hline
\end{tabular}

Fuente: Departament de Cultura de Catalunya, 2017, p.19-20.

Sin embargo, y combinado con ese proceso lento de identificar los bienes de las colecciones, podemos también observar que, a partir de los años 90, con la incorporación de las grandes bases de datos, ha habido un proceso de intento de unificación de formas y fichas de inventario, dado primariamente en los entornos locales y, posteriormente, y a partir de inicios del siglo XXI, este proceso ha derivado a formas intercomunitarias, e incluso, internacionales. Así lo manifestaba Eva Ma Alquézar Yáñez al presentar la base de datos DOMUS, base que integraba distintas colecciones españolas: "La documentación de colecciones, como principal instrumento de protección, se recoge en el Programa de Colecciones del Plan Integral de Museos de la Subdirección General de Museos Estatales (en adelante, SGME) como la primera labor a abordar en orden de prioridad." (ALQUÉZAR, 2004, p. 30). 
Esta integración ha sido sistematizada, especialmente, como resultado de buscar una unidad en las informaciones presentes en el acceso en línea. Sin ser el propósito principal, podría sostenerse que la difusión en línea ha supuesto la promoción de la documentación museográfica, también en los museos pedagógicos. La búsqueda de accesibilidad a las fuentes materiales para su consulta, disfrute o investigación ha conllevado, por lo tanto, un despliegue significativo de los registros, tanto a nivel técnico como informativo. Reyes Carrasco (2012) justamente, argumentaba esta idea en torno la potencialidad de los registros como elemento de partida para la "reutilización" de conocimiento. Una reutilización que, cuando quiere travesar espacios culturales o, incluso, espacios temporales, requiere potenciar la convergencia de registros y vocabularios. Dicha convergencia no es en absoluto fácilmente resoluble en los museos pedagógicos, especialmente, cuando en ellos se han coleccionado bienes comunes que, por propia definición, acumulan en su herencia un lenguaje y unos usos populares, aspecto al cual se añade que, en muchas ocasiones, el registro ha sido realizado por especialistas en historia de la educación -pero no museólogos- o por los mismos docentes de instituciones educativas históricas.

A pesar de estas dificultades, entendemos que la afluencia de nuevas fuentes patrimoniales, como son los bienes inmateriales -especialmente aquellos derivados de la oralidad- o las fuentes audiovisuales, entendidas ya no como complemento, sino como bien patrimonial, nos abre la oportunidad de crear registros museológicos pensados desde la complementariedad de la difusión de la documentación en línea. Una muestra de ello, fue la introducción, en la ficha modelo del Inventari del Patrimoni Etnològic de Catalunya (IPEC), de los nuevos campos: Uso y función del bien; Lugar de uso; Historia y Entrevista, como elementos configurativos de la base de datos con información proveniente de las investigaciones sobre el patrimonio a partir de la historia oral ya sea en entrevistas o conversaciones (COLLELLDEMONT; TORRENTS, 2010).

\section{EL REGISTRO DE LAS PRODUCCIONES AUDIOVISUALES}

Es en el marco de estas ideas de registro unificado, marcos convergentes de fichaje, reutilización y oportunidad ante las nuevas fuentes de estudio patrimonial que, desde el grupo de investigación vinculado al MUVIP ${ }^{2}$, se decidió proceder al registro unificado de las producciones

\footnotetext{
${ }^{2}$ MUVIP: el Museo Virtual de Pedagogía es un centro de documentación y preservación del patrimonio educativo dependiente de la Facultad de Educación, Ciencias Humanas y Traducción de la Universidad de Vic. Se fundó en 2006 con los objetivos de:
}

Rev. Iberoam. Patrim. Histórico-Educativo, Campinas (SP), v. 05, p. 1-18, e019024, 2019. 
audiovisuales realizadas entre 1914 y 1939 (Proyecto $\operatorname{RADAE}^{3}$ ) y, posteriormente, de las producciones audiovisuales de documentales y noticiarios producidos entre 1940 y 1975 (ARAEF ). En ambas ocasiones se procedió a inventariar los audiovisuales accesibles en Filmotecas Públicas. Concretamente en el registro del período entre 1914-1939 pudieron registrarse 142 entradas y del período de 1940-1975, 1538 No-Dos y 370 documentales y reportajes. La diferencia numérica entre ambos registros se explica por la propia evolución histórica de los noticiarios y documentales que derivó en una mayor producción cinematográfica afín al régimen totalitario (RODRÍGUEZ, 1999) y, como detalló Ana Paz (2019) por una mayor preocupación por mostrar la educación deseada en los instrumentos de amplia difusión.

Señalar que la selección se realizó en base a que los documentales o noticiarios tuvieran como eje central la educación, entendida la misma no como escolarización sino como proceso de aprendizaje y enseñanza, que como se puede comprobar, se da en ámbitos tan distintos como la familia, los campamentos, las organizaciones infantiles y juveniles, las prisiones, etc.

1. Ser parte del movimiento que trabaja a favor de la recuperación del patrimonio de la historia de la educación y de la pedagogía.

2. Ofrecer mecanismos de colaboración para la promoción del conocimiento pedagógico y educativo a los estudiantes de educación, y también a los maestros y educadores sociales en activo.

3. Ofrecer un espacio desde el cual canalizar la proyección y creación de innovaciones educativas.

4. Ser plataforma para la visualización de la sociedad educativa presente, ya sea mediante actividades de fórum como a través de exposiciones temporales

5. Crear un espacio para la participación ciudadana, con especial referencia a los maestros, educadores y estudiantes de educación.

(COLLELLDEMONT; PADRÓS en ÁLVAREZ, 2016, p. 146).

Página web: https://www.uvic.cat/es/museo-virtual

${ }^{3}$ RADAE. Revisión y análisis documentales propagandísticos y anuncios audiovisuales educativos producidos entre 1914-1939 en España", Programa Estatal de I+D+I orientada a los Retos de la Sociedad. 2014-2016, (Ref. EDU201348067-R). Acceso registro: https://www.uvic.cat/sites/default/files/documento-en-proceso-registro-films.pdf

${ }^{4}$ ARAEF. Análisis de las representaciones audiovisuales de la educación en documentales y noticiarios durante el franquismo. Programa Estatal de Investigación, Desarrollo e Innovación Orientada a los Retos de la Sociedad, en el marco del Plan Estatal de Investigación Científica y Técnica y de Innovación 2013-2016, (Ref. EDU2017-89646-R, AEI/FEDER, UE). Acceso registro: http://hdl.handle.net/10854/5845

Rev. Iberoam. Patrim. Histórico-Educativo, Campinas (SP), v. 05, p. 1-18, e019024, 2019. 
Tablas 2 y 3- Fragmentos de los registros RADAE y ARAEF realizados por el equipo del MUVIP.

\begin{tabular}{|c|c|c|c|c|c|c|}
\hline "ृष & 总 & 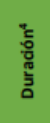 & 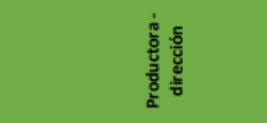 & 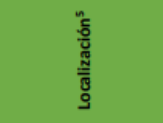 & 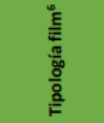 & 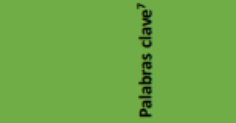 \\
\hline $\begin{array}{l}1912-1920 \\
\text { ap. }\end{array}$ & Una visita a la isla de los niños & $110^{\prime}$ & $\begin{array}{l}\text { Prod: Antonio de P. Tramullas } \\
\text { Dir.: Antonio de Pádua Tramullas }\end{array}$ & Filmoteca Española & $\begin{array}{l}\text { Documental } \\
\text { - reportaje }\end{array}$ & $\begin{array}{l}\text { Sanatorio. } \\
\text { Hospitales infantiles. }\end{array}$ \\
\hline 1914 & Primera comunión de Mercedes Curá Roxio & $284^{\prime}$ & $\begin{array}{l}\text { Prod: Doméstica } \\
\text { Dir.: - }\end{array}$ & Filmoteca Española & Documental & Comunión en escuela. \\
\hline 1914 & Logroño: sus panoramas y bellezas & $6^{\prime}$ & $\begin{array}{l}\text { Prod.: Casa Tramullas } \\
\text { Dir.: Antonio de Pádua Tramullas }\end{array}$ & Filmoteca Española & Documental & Escultismo. \\
\hline 1915-1925 & [Los exploradores de España - Fiesta de San Jorge] & $\cdot$ & $\begin{array}{l}\text { Prod: Sallumart Films } \\
\text { Dir.: Antonio de Pádua Tramullas }\end{array}$ & Filmoteca Española & $\begin{array}{l}\text { Documental } \\
\text { - reportaje }\end{array}$ & $\begin{array}{l}\text { Escultismo. } \\
\text { Educación fisica. Autoridades. }\end{array}$ \\
\hline $\begin{array}{l}\text { 1916-1920 } \\
\text { ap. }\end{array}$ & [Niños exploradores de Zaragoza. Ejercicios-Juegos] & $\cdot$ & $\begin{array}{l}\text { Prod.: Sallumart Films } \\
\text { Dir.: Antonio de Pádua Tramullas }\end{array}$ & Filmoteca Española & $\begin{array}{l}\text { Documental } \\
\text { - reportaje }\end{array}$ & $\begin{array}{l}\text { Escultismo. } \\
\text { Educación fisica. }\end{array}$ \\
\hline 1916 & $\begin{array}{l}\text { Zaragoza. Año 1916. Detalles de la Fiesta del árbol } \\
\text { celebrada en 1. De abril }\end{array}$ & - & $\begin{array}{l}\text { Prod: Sallumart Films } \\
\text { Dir.: Antonio de Pádua Tramullas }\end{array}$ & Filmoteca Española & Reportaje & $\begin{array}{l}\text { Autoridades. } \\
\text { Alumnado. }\end{array}$ \\
\hline
\end{tabular}

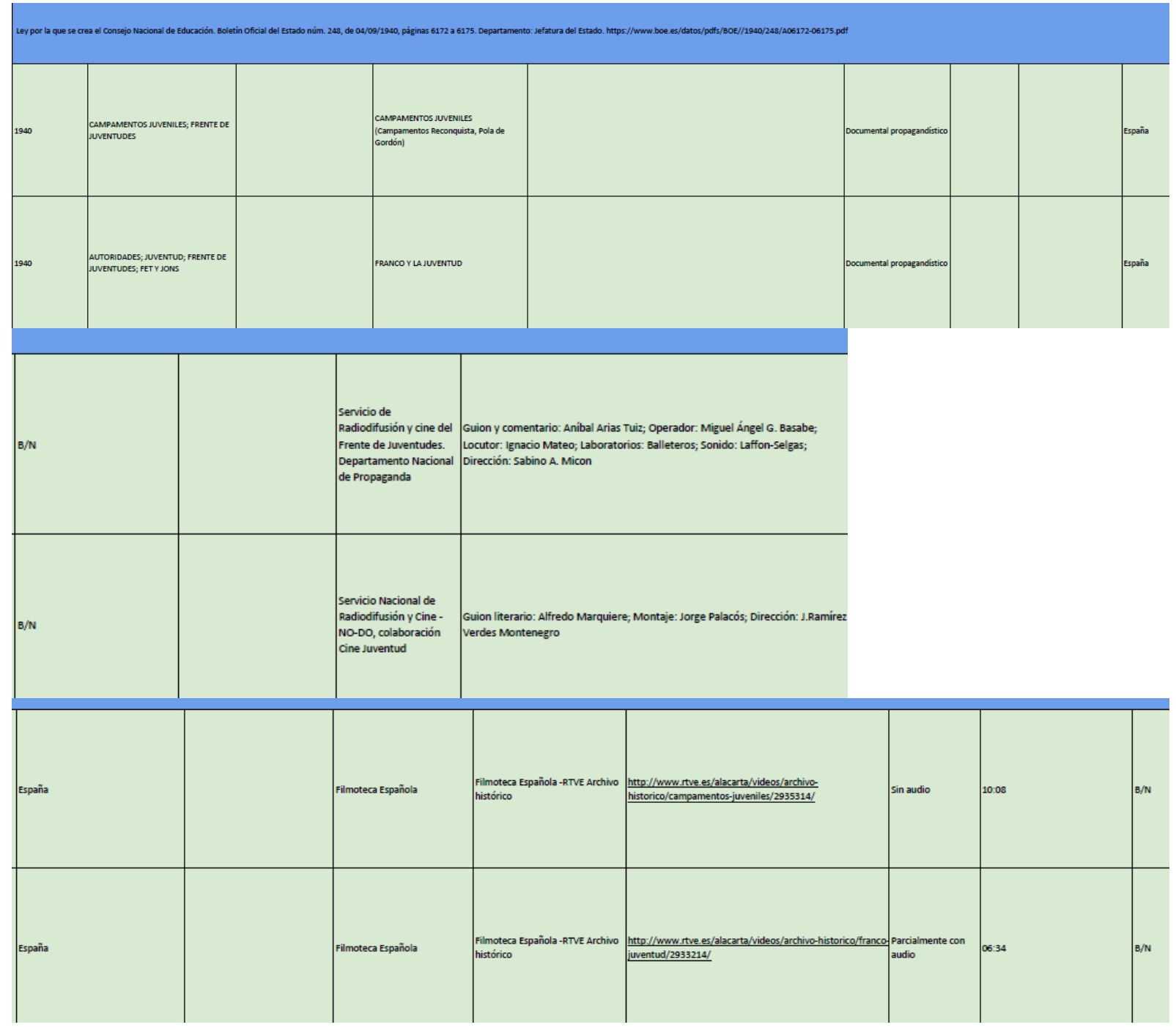

Fuente: MUVIP.

En ambos proyectos, aquello que se pretendía era poner a disposición de las personas interesadas en el patrimonio histórico educativo (investigadores, estudiantes de las facultades de

Rev. Iberoam. Patrim. Histórico-Educativo, Campinas (SP), v. 05, p. 1-18, e019024, 2019. 
educación, profesorado...) una base de datos de referencias amplias sobre un material patrimonial que está adquiriendo cada vez más relevancia en el ámbito histórico-educativo (Paranaguá, 2003; Warmington, Van Gorp, Grosvenor, 2011; Galak y Orbuch, 2017; Duran y Álvarez, 2018) como se destacó en el Seminario From propaganda to resistance. Vital options in the totalitarianism, celebrado en Vic del 2 al 4 de mayo de 2019.

A pesar de compartir esta finalidad, y como se puede comprobar visualmente en la reproducción parcial de la lista, los campos de registro utilizados en los dos proyectos han divergido un poco, puesto que en el último proyecto consideramos necesario ampliar la ficha para facilitar el acceso al patrimonio ya digitalizado y mejorar la información registrada en los ámbitos técnicos de producción, tal y como se puede observar en la tabla comparativa siguiente:

Tabla 4 - Ficha de campos registrados según el proyecto.

\begin{tabular}{|l|l|}
\hline Campos proyecto RADAE & Campos proyecto ARAEF \\
\hline Año & Año \\
\hline Palabra clave & PALABRAS CLAVE (y alertas) \\
\hline \multirow{4}{*}{ Título } & Nombre serie \\
\cline { 2 - 2 } & Título o nombre propio \\
\cline { 2 - 2 } & Subtítulo \\
\hline \multirow{2}{*}{ Datación } & Fecha \\
\cline { 2 - 2 } & Observaciones a la datación \\
\hline \multirow{2}{*}{ Uuración } & Lugar de producción \\
\hline \multirow{5}{*}{ Productora - dirección } & Características técnicas: duración \\
\cline { 2 - 2 } & Características técnicas: soporte \\
\cline { 2 - 2 } & Características técnicas: música \\
\hline \multirow{5}{*}{ Localización } & Fuente de financiación y dependencia \\
& orgánica \\
\cline { 2 - 2 } & Productora \\
\cline { 2 - 2 } & Equipo de dirección y realización \\
\hline Tipología film & Institución de conservación \\
\cline { 2 - 2 } & Localización de ejemplares \\
\cline { 2 - 2 } & Acceso Enlace \\
\hline & Tipología de film \\
\hline & Comentarios y entrevistas \\
\hline
\end{tabular}

Fuente: Elaboración propia a partir de los registros.

Una de las cuestiones que fueron más complejas de la elaboración del registro fue la correspondiente a lo que podríamos denominar como campos analíticos e interpretativos. Estos son los correspondientes a las palabras clave y a la tipología de film. Como se desarrolla en los

Rev. Iberoam. Patrim. Histórico-Educativo, Campinas (SP), v. 05, p. 1-18, e019024, 2019. 
materiales: Registro de los reportajes y noticias en los que aparece representada la educación (1940-1975), la cuestión de las palabras clave se resolvió siguiendo un proceso inductivo analítico que consistió en el vaciado de los títulos del registro y agrupación según las siguientes dimensiones:

IDEOLOGÍAS Y SITUACIONES POLÍTICAS
PROPÓSITO EDUCATIVO
INSTITUCIONES / ENTORNOS EDUCATIVOS
GRADOS EDUCATIVOS
ÁREAS EDUCATIVAS Y DE CONOCIMIENTO
COMUNIDAD EDUCATIVA
PROCESOS EDUCATIVOS
PROTECCIÓN INFANCIA
MODELOS VITALES
SUCESOS
ÁMBITO GEOGRÁFICO

Por otra parte, otra tarea que nos pareció importante fue la de procurar agrupar los grupos semánticos buscando, por una parte, la coherencia con la etapa estudiada y, por otra, facilitar la lectura desde la comprensión y conceptualización actual de la educación. Un buen ejemplo de esta agrupación es el que señalamos en el texto de presentación del registro:

Un ejemplo de estas agrupaciones es la identificación de "centros para la primera infancia" de aquellos denominados de las siguientes formas: casa cuna / casacuna; casa de maternidad; casa del niño; colonia infantil; educación preescolar; guardería infantil; guardería jardín maternal; guarderías; hogar de la madre y del niño; hogar infantil; hogar-cuna; jardín de (la) infancia; jardín de niños; jardín infantil; jardín maternal. (COLLELLDEMONT, 2019, p. 3).

En cuanto al análisis de las tipologías de audiovisuales se establecieron dos grupos:

Tipología de formato:

- documental

- noticiario

- documental-noticiario

Tipología de contenido:

- propagandístico

- resistencia 
A través de los diferentes seminarios celebrados con motivo de discutir esta clasificación se consideró que los reportajes de los $\mathrm{NO}-\mathrm{DO}^{5}$ se correspondían siempre con la tipología de documental-noticiario con contenidos propagandísticos, mientras que los documentales clandestinos (producidos al margen de la oficialidad de la dictadura) eran documentales de tipo de propaganda institucional no oficial o de resistencia.

\section{MÁS ALLÁ DEL REGISTRO}

Así pues, en el ámbito del estudio de la memoria educativa ${ }^{6}$, los inventarios permiten cumplir con las funciones de conservación, difusión e investigación sobre el patrimonio histórico educativo (MENEZES, 2011; BRAGHINI et al., 2016). En este sentido, nuestro grupo ha empezado ya, a través del registro, algunas investigaciones con el patrimonio audiovisual inventariado del proyecto ARAEF relacionadas con la práctica escolar proyectada en las noticias ${ }^{7}$, la enseñanza universitaria que muestra el noticiario ${ }^{8}$, los campamentos infantiles y juveniles durante el franquismo ${ }^{9}$, la representación de la sección femenina ${ }^{10}$ y, también ha sido utilizado por

${ }^{5}$ NO-DO, Noticiarios y Documentales Cinematográficos, era una publicación audiovisual de carácter de noticiario propagandístico del franquismo, centralizado, único y obligatorio. El primer NO-DO se proyectó por primera vez en las pantallas de los cines españoles el 4 de enero de 1943 y desde su creación hasta su cierre, en 1976, un año después de la muerte del dictador Francisco Franco, fue obligatoria su proyección en todos los locales de exhibición cinematográfica (TRANCHE; SÁNCHEZ-BIOSCA, 1993).

${ }^{6}$ Señalamos como ejemplo de actualidad el trabajo realizado por el grupo de historiadores de Manresa: memoria.cat que están poniendo a disposición los distintos inventarios como eje para poder informar los testimonios orales. Los resultados pueden consultarse en: http://www.memoria.cat/mestres . Consideramos que el mismo es un ejemplo de trabajo realizado desde la perspectiva de la "historia pública" en los que el profesorado académico trabaja conjuntamente con la ciudadanía.

${ }^{7}$ Los resultados se presentaron en: JORNADAS CIENTÍFICAS DE LA SEPHE, 2018: VIII Jornadas Científicas de la SEPHE. La Práctica Educativa. Mallorca del 20 al 23 de novembre de 2018. Pizarra y pupitre. La representación de la práctica escolar a través del NO-DO en las inauguraciones de escuelas durante franquismo: Josep Casanovas i Núria Padrós; JORNADES D'HISTÒRIA DE L'EDUCACIÓ, 2018 XXIII Jornades d'Història de l'Educació. Educació i desenvolupament rural als segles XIX i XX. Lleida del 25 al 27 d'octubre de 2018.El franquisme i l'ensenyament agrícola a Catalunya a través del NO-DO. Una visita en blanc i negre a la granja escola de Caldes de Montbui: Josep Casanovas , Raquel Cercós i Jordi Garcia.

${ }^{8}$ Los resultados se presentaron en: COL·LOQUI HELOÏSE, 2019 Col.loqui Internacional Heloïse. Pedagogies de la resistència i de la democràcia. IEC. Barcelona del 7 al 9 de febrer de 2019. La història de l'educació a Catalunya: lluites, resistència i democràcia. Els cinc itineraris proposats a Catalunya: Conrad Vilanou; Les pedagogies de la resistència en els espais urbans representats en dictadures: Eulàlia Collelldemont y Raquel Cercós.

${ }^{9}$ Los resultados se presentaron en: ISCHE 40, ISCHE 40. Education and Nature. Humboldt University, Berlin, 2018: Preformed panel: Nature as a propagandistic icon during totalitarianisms. Tracing transnational semblances and differences showed in visual representations. (Eulàlia Collelldemont, Raquel Cercós, Ian Grosvenor, Iveta Kestere, Anne Bruch).

${ }^{10}$ Los resultados se presentaron en: ISCHE 41, ISCHE 41. Spaces and places of education, Universidade de Porto, 2019. Representaciones de la Sección Femenina en los espacios públicos: pluralidades y paradojas de género Pilar Prat, Isabel Carrillo.

Rev. Iberoam. Patrim. Histórico-Educativo, Campinas (SP), v. 05, p. 1-18, e019024, 2019. 
estudiantes de nuestra universidad para realizar sus trabajos de fin de grado, en los que se analiza un aspecto concreto desarrollado en clave de evolución temporal a partir del análisis tanto de contenidos como de imágenes ${ }^{11}$.

Pero más allá de dichos usos, el mismo registro nos ha permitido realizar estudios seriados de aspectos educativos concretos, que, también nos aportan información relevante sobre qué dimensiones y temas aparecían más en los documentales y noticiarios inventariados.

Para llevar a cabo el estudio de las series registradas, hemos realizado un análisis cuantitativo de la frecuencia en la que aparecen las distintas dimensiones en las que se hallan agrupadas las palabras claves utilizadas en el registro del proyecto. Presentamos a continuación los gráficos de dichas frecuencias calculadas en función de la tipología de formato (Gráfico 1documentales educativos y Gráfico 2 - documentales-noticiarios). Hemos realizado gráficos de rectángulos que permiten visualizar los datos cuantitativos de las distintas dimensiones recogidas jerárquicamente y, además, se presentan, para las tres dimensiones que más han aparecido en cada tipología, un gráfico de barras, con la información de la frecuencia de cada palabra clave:

\footnotetext{
${ }^{11}$ Como ejemplo citamos el trabajo de FÀBREGA, Sara. Concepció del rol de la dona durant l'època franquista. Trabajo Final de Grado. Vic: Universitat de Vic, 2019. Disponible en: http://dspace.uvic.cat/xmlui/handle/10854/5944. Acceso: 12 nov. 2019.
} 
Gráfico 1-Frecuencias de las dimensiones y palabras clave en los documentales educativos.
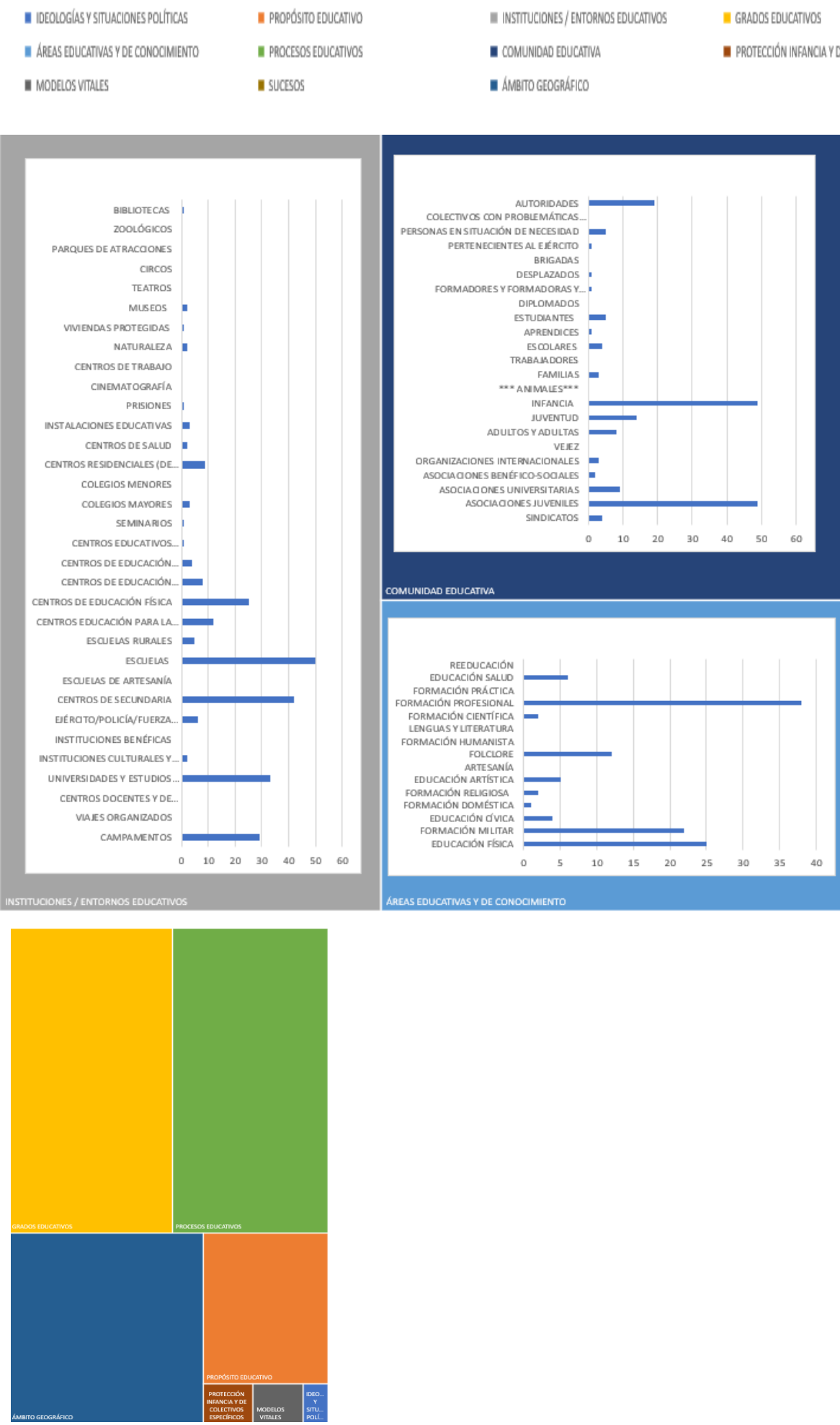

Fuente: Elaboración propia

Rev. Iberoam. Patrim. Histórico-Educativo, Campinas (SP), v. 05, p. 1-18, e019024, 2019. 
Gráfico 2-Frecuencias de las dimensiones y palabras clave en los documentales-noticiarios.
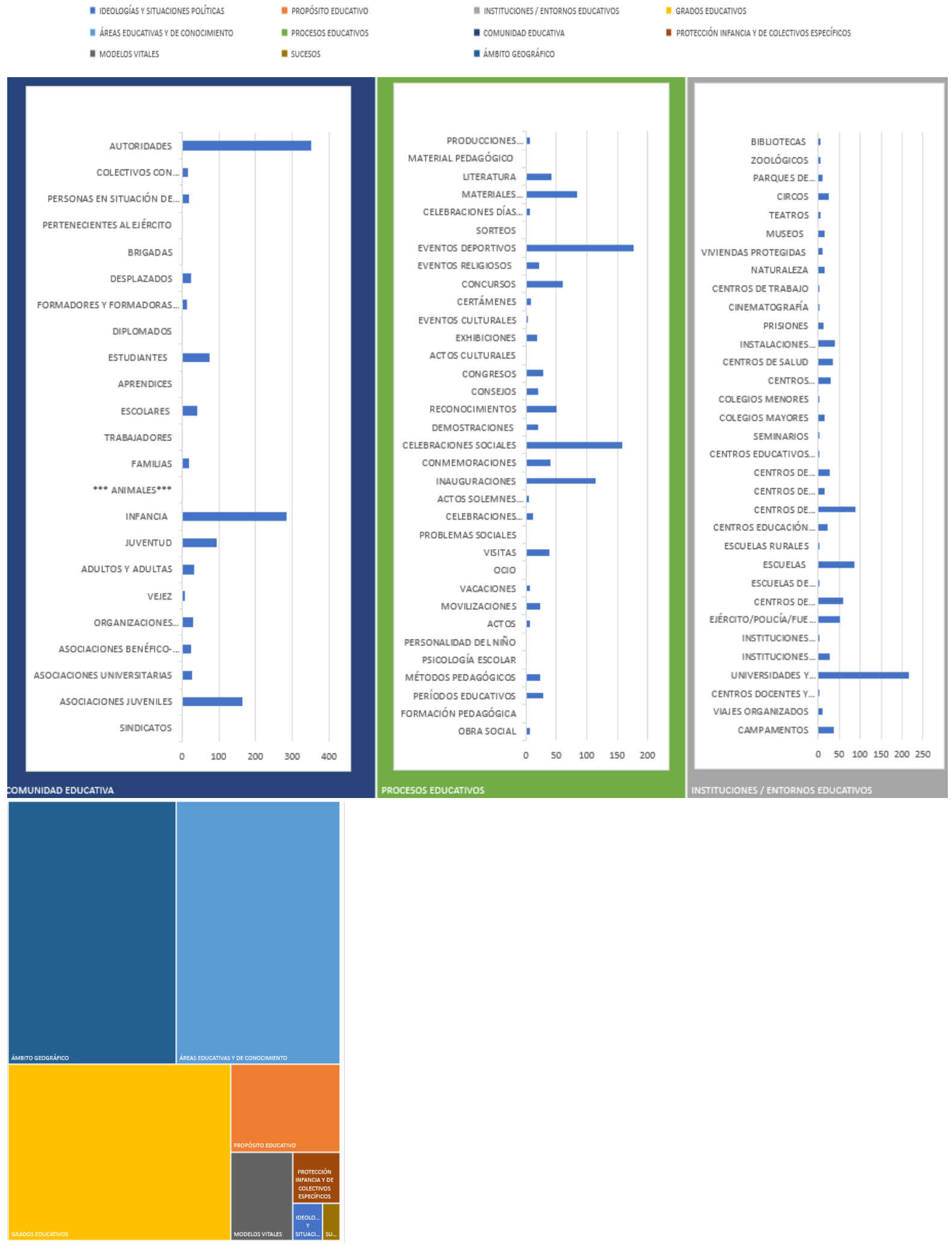

Fuente: Elaboración propia.

Rev. Iberoam. Patrim. Histórico-Educativo, Campinas (SP), v. 05, p. 1-18, e019024, 2019. 
En los gráficos anteriores se observa que las dimensiones que más aparecen en los documentales registrados, son, en primer lugar, las instituciones y entornos educativos, comunidad educativa y áreas educativas y de conocimiento, mientras que en los noticiarios-documentales comunidad educativa es la que se encuentra con mayor frecuencia, seguida de procesos educativos y de instituciones educativas. Esta primera aproximación nos permite entrever, pues, diferencias entre los documentales y los documentales-noticiarios en cuanto a los aspectos que se querían resaltar en cada tipología de film (JURISTO, 2008).

Por otra parte, si entramos en las palabras claves, vemos, también diferencias significativas, como por ejemplo la alta presencia de autoridades en los documentales-noticiarios (en los documentales, aunque presentes, es mucho menor), la menor representación de la escuela en los noticiarios-documentales que no en los documentales, o la alta representación de la universidad en los noticiarios-documentales, seguramente debido al prestigio de la educación superior o a la necesidad de reforzar la idea que el progreso del país se debía al trabajo de los científicos (TABERNERO, 2008).

Otro aspecto que llama la atención son las frecuencias relacionadas con las áreas educativas. Concretamente, cabe destacar la enorme representación de la formación profesional en ambas tipologías de audiovisuales, aspecto que es coherente con la imagen del ciudadano que tenía como objetivo máximo el trabajo, así como con la voluntad de hacer manifiesto el principio franquista de la acción por la acción como premisa básica (ECO, 2018, 39).

Asimismo, otro elemento relevante dentro de las áreas educativas, es la presencia notable de la educación artística en ambas tipologías, especialmente si la comparamos, por ejemplo, con la formación humanista (que aparece muy poco representada). De hecho, la poca representación de la educación humanista nos ha sorprendido, puesto que el imaginario habitual sobre la educación en el franquismo es que a la educación humanista tenía más importancia que a la artística o incluso a la científica (que también aparece más representada en ambas tipologías). Este resultado inesperado es uno de los que nos hemos propuesto abordar para intentar dar una explicación a esta discrepancia.

También es destacable la elevada frecuencia con la que aparecen tanto la educación militar como la educación física en ambas tipologías de films, aunque este resultado era, quizá, más previsible teniendo en cuenta el contexto político totalitario (derivado de un golpe militar) en el que se realizaron las producciones audiovisuales estudiadas, así como la finalidad propagandística con la que fueron elaboradas (TRANCHE; SÁNCHEZ BIOSCA, 2006). 
En contraposición, la formación religiosa aparece en los documentales inventariados, pero con una frecuencia bastante baja. A priori, este resultado podría resultar sorprendente en el contexto del nacionalcatolicismo español de la época, pero cabe destacar que, aunque no aparece la formación religiosa, sí que abundan imágenes de personal religioso (autoridades, curas, monjas, ...) ejerciendo distintas tareas. El totalitarismo religioso queda patente en estas imágenes: la religión estaba en todos los ámbitos, en todos los momentos de la vida reflejados en las producciones audiovisuales, y quizá por eso, ya no era necesario resaltar la formación religiosa.

\section{CONCLUSIONES Y PERSPECTIVAS}

Como se em ido indicando a lo largo del texto, desde el grupo del MUVIP se em considerado que los registros son dispositivos facilitadores de las investigaciones, posibilitando em ordenación cronológica de los materiales preservados, así como la posibilidad de construir bases de datos públicas y a disposición de las personas interesadas em esta tipología de patrimonio. Es por ello que podemos afirmar que em la digitalización de los mismos no solamente se desarrolla em mecanismo de gestión, sino que además se promueve la creación de herramientas para la visualizar em determinado tipo de patrimonio histórico educativo. Em este sentido, entendemos que se explora la virtualidad em su posibilidad de dar valor para la investigación histórico educativo a los documentales y noticiarios que, em em determinado período, fueron los dispositivos fundamentales de propaganda del régimen, de sus políticas - y entre las mismas la educativa- y de sus valores. Em coherencia em ello, el poder atender a estos materiales em forma de series, posibilita la visualización de las temáticas priorizadas, poniendo de relieve aquellos tópicos más utilizados como elemento de propaganda, siendo algunos de los mismos conocidos, habiendo pasado otros desapercibidos em los estudios histórico-temáticos como lo es educación artística o la apropiación de modelos educativos extranjeros (ambos aspectos de constante aparición em pantalla).

Por lo tanto, concluiríamos que los registros realizado em el marco de nuestros proyectos, más allá de cumplir em las funciones anteriormente señaladas, también nos em permitido confirmar algunas sospechas -como por ejemplo el uso del edificio escolar como decorado para la puesta em escena de las autoridades- y romper estereotipos -como sería el papel activo de la mujer em el marco de la Sección femenina o la importancia otorgada a la educación profesional-. Nos han

Rev. Iberoam. Patrim. Histórico-Educativo, Campinas (SP), v. 05, p. 1-18, e019024, 2019. 
provocado preguntas - como serían las correspondientes al perfil de estudiante universitario, como se resolvían las disonancias entre el papel de figurante de las mujeres y el papel predominante de las mismas em las organizaciones femeninas, como se integraban las experiencias de educación del extranjero mostradas em em entorno de exclusión de lo ajeno, etc. y nos han posibilitado recoger indicadores sobre como se han construido los imaginarios educativos de nuestro entorno sobre el orden, la disciplina, la práctica, la reeducación, etc. Em este sentido, creemos que estamos em situación de poder afirmar que los registros son em herramienta muy potente para los expertos del ámbito del patrimonio histórico educativo y que su elaboración permite mejorar los estudios, por cuanto pone de relieve los aspectos que, por su alta presencia o bien por omisión, interrogan a los investigadores, deviniendo em ello em herramienta que posibilita el desarrollo de investigaciones em continuo que se entrelacen y permitan avanzar em el establecimiento de datos basales.

\section{BIBLIOGRAFÍA}

ALQUÉZAR, Eva M. ${ }^{a}$ Domus, un sistema de documentación de museos informatizado. Estado de la cuestión y perspectivas de futuro. Museos.es: Revista de la Subdirección General de Museos Estatales, n. 0, p. 28-41, 2004.

ÁLVAREZ DOMÍNGUEZ, Pablo (coord.). Los museos pedagógicos en España. Entre la memoria y la creatividad. Gijón, Ediciones Trea, 2016, 260.

BRAGHINI, Katia Zuquim et al. Apresentação da ficha de inventário e história do Museu Escolar do Colégio Marista Arquidiocesano de São Paulo. RIDPHE_R Revista Iberoamericana do Patrimônio Histórico-Educativo, v. 2, n. 2, p. 73-91, 27 jun. 2016.

CARRASCO GARRIDO, Reyes. Documentar el patrimonio: cuando la información se transforma en un recurso sostenible. Museos.es: Revista de la Subdirección General de Museos Estatales, n. 7-8, p. 120-125, 2012.

COLLELLDEMONT, Eulàlia (coord.) Registro de los reportajes y noticias del NO-DO en los que aparece representada la educación (1940-1975), Vic, MUVIP, 2019, 158. Disponible en: http://hdl.handle.net/10854/5845. Acceso: 17 jun. 2019.

COLLELLDEMONT, Eulàlia; TORRENTS, Jacint. Inventari i documentació del patrimoni educatiu de les escoles de la comarca d'Osona. El CEIP El Roure Gros de Santa Eulàlia de Riuprimer. Revista d'etnologia de Catalunya, n. 35, p. 212-216, 2010. 
DEPARTAMENT DE CULTURA. Museus 2030. Pla de Museus de Catalunya. Barcelona: Generalitat de Catalunya, 2017, 276. Disponible en:

https://cultura.gencat.cat/web/.content/sscc/pla-museus-2030/documents/PMC_web.pdf. Acceso: 17 jun. 2019.

DURAN, Valeriano; ÁLVAREZ, Pablo. La imagen de la escuela en la primera etapa del cine español del franquismo: autarquía, patriotismo y nacionalcatolocismo (1939-1950). Educació i Història, n. 31, p. 59-88, 2018.

ECO, Umberto. Contra el fascismo. Barcelona: Lumen, 2018.

GALAK, Eduardo; ORBUCH, Iván, Cine, educación y cine educativo en el primer peronismo. El caso del Departamento de Radioenseñanza y Cinematografía Escolar. Cine documental, n.16, 2017. Disponible en: http://revista.cinedocumental.com.ar/cine-educacion-y-cine-educativo-enel-primer-peronismo-el-caso-del-departamento-de-radioensenanza-y-cinematografia-escolar/. Acceso: 11 nov. 2019.

JURISTO, Álvaro Matud. La incorporación del cine documental al proyecto de NO-DO. Historia y comunicación social n.13, p.105-118, 2008.

MENEZES, Maria Cristina. Descrever os documentos - construir o inventário - preservar a cultura material escolar. Revista Brasileira de História da Educação, Campinas-SP, v.11, n.1 (25), p. 93-116, ene./abr. 2011.

MUVIP. Disponible en: https://www.uvic.cat/sites/default/files/documento-en-proceso-registrofilms.pdf.

MUVIP. Disponible en: https://www.uvic.cat/sites/default/files/registro_repnot.pdf.

PARANAGUÁ, Paulo Antonio. El cine documental en América Latina. Madrid: Cátedra.

PAZ, Ana. To legitimise the Dictatorship through the Film makers. Conceptual challenges. 2019. Disponible en: https://mon.uvic.cat/araef-conceptual-challenges/2019/05/21/ana-paz-tolegitimise-the-dictatorship-through-the-film-makers/. Acceso: 11 nov. 2019.

RODRÍGUEZ MARTÍNEZ, Saturnino. NO-DO, catecismo social de una época. Madrid, Editorial Complutense, 1999.

TABERNERO, Carlos. The Changing Nature of Modernization Discourses in Documentary Films. Science in context. n. 31.1 p. 61-83, 2018.

TRANCHE, Rafael R.; SÁNCHEZ-BIOSCA, Vicente. NO-DO: entre el desfile militar y la foto de família. Archivos de la Filmoteca, n. 15, p. 41-53, 1993.

TRANCHE, Rafael R.; SÁNCHEZ-BIOSCA, Vicente. NO-DO. El tiempo y la memoria. Madrid, Ediciones Cátedra, Filmoteca Española, p. 257-259, 2006. 
WARMINGTON, Paul, VAN GORP, Angelo; GROSVENOR, Ian. Education in motion: uses of documentary film in educational research. Paedagogica Historica: International Journal of the History of Education, n. 47:4, p. 457-472, 2011.

Recebido em: 27 de junho de 2019

Aceito em: 16 de novembro de 2019 